\title{
Short-Term Depression of Synaptic Transmission during Stimulation in the Globus Pallidus of 1-Methyl-4-Phenyl- 1,2,3,6-Tetrahydropyridine-Treated Primates
}

\author{
Yaara Erez, ${ }^{1}$ Hadass Czitron, ${ }^{1}$ Kevin McCairn, ${ }^{1}$ Katya Belelovsky, ${ }^{1}$ and Izhar Bar-Gad ${ }^{1,2}$ \\ ${ }^{1}$ Gonda Brain Research Center, and ${ }^{2}$ Goodman Faculty of Life Sciences, Bar-Ilan University, Ramat Gan 52900, Israel
}

High-frequency stimulation (HFS) in the globus pallidus is used to ameliorate clinical symptoms of Parkinson's disease, dystonia, and other disorders. Previous in vivo studies have shown diverse static effects of stimulation on discharge rates and firing patterns of neurons along the corticobasal ganglia loop. In vitro studies, together with other experimental and theoretical studies, have suggested the involvement of synaptic plasticity in stimulation effects. To explore the effects of HFS on synaptic transmission, we studied the dynamic changes in neuronal activity in vivo, using multielectrode recordings during stimulation in the globus pallidus of 1-methyl-4-phenyl-1,2,3,6tetrahydropyridine (MPTP)-treated primates. Stimulation effects evolved over time and were pronounced during the first $10 \mathrm{~s}$ of stimulation, where $69 \%$ of the 249 recorded neurons changed their firing rate and $61 \%$ displayed time-locked firing. The time-locked response faded away in $43 \%$ of the responding neurons, and its pattern was altered in the remaining cells: the peak response shifted away in time from the stimulus onset, and its amplitude decreased. Repetition of the stimulation protocol revealed a full resetting of the effect, implying short-term synaptic depression. This evolving response is indicative of the transient plasticity of the corticobasal ganglia network in vivo during HFS. Therefore, short-term depression of synaptic transmission may contribute to the mechanism underlying the effects of stimulation during the resulting steady state, altering the balance of neuronal interactions and interfering with pathological information transmission.

\section{Introduction}

High-frequency electrical stimulation (HFS) in deep brain structures, which are part of the corticobasal ganglia (CBG) loop such as the globus pallidus (GP), is a commonly used treatment for Parkinson's disease (PD) (Rodriguez-Oroz et al., 2005), dystonia, Tourette syndrome, and other disorders (Halpern et al., 2007). Despite the therapeutic benefits of HFS, its effects on underlying neuronal activity are still controversial.

Multiple studies have documented the modulation of both discharge rates and firing patterns during and after HFS throughout the CBG loop. Early studies reported inhibition of neuronal activity within the stimulated nucleus during HFS in the globus pallidus internal segment (GPi) (Dostrovsky et al., 2000) and subthalamic nucleus (STN) (Filali et al., 2004) of humans and the GPi of primates (Boraud et al., 1996). Later studies focused on changes in the temporal pattern of neuronal firing during stimulation. HFS resulted in finely timed changes in firing with latencies as short as a few milliseconds in the primate GP during STN (Hashimoto et al., 2003; Kita et al., 2005) and GP (Bar-Gad et al.,

\footnotetext{
Received Jan. 26, 2009; revised May 3, 2009; accepted May 12, 2009.

This study was supported in part by Israel Science Foundation (ISF) Grant 1000-05 and ISF Converging Technologies Grant 1698-07. We thank Dr. N. Nagorski for providing technical support, Dr. M. Dror and P. Malamud for animal care, A. Tzameret for animal care and recording assistance, and Dr. A. Korngreen and Dr. D. Cohen for fruitful discussions.

Correspondence should be addressed to Izhar Bar-Gad, Gonda Brain Research Center, Bar-llan University, Ramat Gan 52900, Israel. E-mail: bargadi@mail.biu.ac.il.

DOI:10.1523/JNEUROSCI.0401-09.2009

Copyright $\odot 2009$ Society for Neuroscience $\quad$ 0270-6474/09/297797-06\$15.00/0
}

2004; McCairn and Turner, 2009) stimulation, and in the thalamus during STN (Xu et al., 2008) and GPi (Montgomery, 2006) stimulation. Furthermore, HFS was shown to affect other firing pattern properties including oscillatory and bursty activity (Meissner et al., 2005; Montgomery, 2006; Dorval et al., 2008; Hahn et al., 2008; Xu et al., 2008; McCairn and Turner, 2009).

The accumulating evidence highlights the importance of both firing rate and pattern changes during stimulation. These studies, however, assumed static stimulation effects over time. In vitro studies have demonstrated that GP stimulation induces evolving effects by modulation of synaptic activity through a mechanism of short-term plasticity (Hanson and Jaeger, 2002; Rav-Acha et al., 2005). It was recently shown that synaptic plasticity is enhanced in PD patients during deep brain stimulation (DBS) (Prescott et al., 2009). Other theoretical and experimental studies have suggested the existence of synaptic plasticity during HFS (McIntyre et al., 2004; McCairn and Turner, 2009). These findings led us to hypothesize that synaptic plasticity is involved in altering the steady state of information transmission that underlies the effects of HFS on PD. Thus, the aim of this study is to explore in vivo the dynamics of GP-HFS effects on pallidal neuronal activity in the parkinsonian primate.

\section{Materials and Methods}

Animals. Two cynomolgus (Macaca fascicularis) male monkeys were used (A: $3.7 \mathrm{~kg}$; N: $4 \mathrm{~kg}$ ). The monkeys' water and food consumption and weight were followed daily, and their health was monitored by a veterinarian. All procedures were in accordance with the National Institutes of 
Health Guide for the Care and Use of Laboratory Animals and Bar-Ilan University Guidelines for the Use and Care of Laboratory Animals in Research and were approved and supervised by the Institutional Animal Care and Use Committee.

Surgery and induction of parkinsonism. The monkeys underwent a surgical procedure to attach a $27 \mathrm{~mm}$ square Cilux recording chamber (Alpha Omega Engineering) to the skull allowing access to the GP. The chamber was tilted $40^{\circ}$ in the sagittal plane, with its center targeted at stereotaxic coordinates A12-L7-H14 (Szabo and Cowan, 1984) of the right hemisphere for monkey A, and A13-L8-H13 of the left hemisphere for monkey $\mathrm{N}$. The surgical procedure was performed under general anesthesia [induced by intramuscular ketamine- $\mathrm{HCl}(10 \mathrm{mg} / \mathrm{kg})$ and Domitor $(0.1 \mathrm{mg} / \mathrm{kg})$ and maintained by isoflurane and $\mathrm{N}_{2} \mathrm{O}$ ventilation] and aseptic conditions. Parkinsonism was induced by five intramuscular injections of $0.4 \mathrm{mg} / \mathrm{kg}$ 1-methyl-4-phenyl-1,2,3,6-tetrahydropyridine (MPTP)-HCl (Sigma) neurotoxin. MPTP injections were given under intramuscular ketamine- $\mathrm{HCl}(10 \mathrm{mg} / \mathrm{kg})$ anesthesia and over a period of $4 \mathrm{~d}$, after which the monkeys developed severe parkinsonism. The symptoms included all the main parkinsonian symptoms except for rest tremor, which is typically not exhibited by this species. Additionally, both monkeys had dystonia, primarily in the lower limbs. The monkeys' parkinsonian state was assessed daily using the Schneider scale (Schneider et al., 2003) and was severe and stable throughout the recording period (mean \pm SD: monkey A: $45.75 \pm 3.37$, monkey N: $46.58 \pm 2.71$, scale of 0 (asymptomatic) to 53 (maximal symptoms). Recordings were resumed 4 and $5 \mathrm{~d}$ after the last injection for monkeys $\mathrm{A}$ and $\mathrm{N}$, respectively.

Recording and stimulation. The monkeys were seated in a primate chair and their head was fixed during the recording sessions. Using a cylindrical guide (1.7/2.15 mm inner/outer diameter), eight glass-coated tungsten microelectrodes (impedance $0.2-0.7 \mathrm{M} \Omega$ at $1 \mathrm{kHz}$ ) were advanced separately (EPS 4.10, Alpha Omega Engineering) into the GP. The electrode signal was continuously sampled at $40 \mathrm{kHz}$ (Alphamap 10.10, Alpha Omega Engineering), amplified $\left({ }^{*} 1000\right)$, and bandpass filtered $(2-$ $8000 \mathrm{~Hz}$ four-pole Butterworth filter) (MCP-Plus 4.10, Alpha Omega Engineering). The distinction between the external (GPe) and internal (GPi) segments was determined online based on characteristics of neuronal activity (oscillations, background noise, etc.), and the existence of border cells and white matter fibers between the two segments. Border cells were excluded from this study, and most of the recorded neurons in the GPe were high-frequency pausers and not low-frequency bursters.

High-frequency monopolar microstimulation was delivered using one of the eight electrodes (impedance $0.2-0.35 \mathrm{M} \Omega$ at $1 \mathrm{kHz}$ ). The stimulation pulses consisted of $40 \mu \mathrm{A}$ biphasic currents (200 $\mu$ s cathodal followed by $200 \mu$ s anodal phase) (see supplemental material 2, available at www.jneurosci.org, for details). The interval between consecutive pulses was $7 \mathrm{~ms}$, leading to a stimulation frequency of $135.1 \mathrm{~Hz}$ for $37 \mathrm{~s}(5000$ stimuli) and $126 \mathrm{~s}$ (17,000 stimuli) for monkeys A and N, respectively. Some of the neurons were recorded during a second stimulation session for $30 \mathrm{~s}$ (4000 stimuli).

Data analysis and stimulation artifact removal. The digitized continuous signal of each electrode was preprocessed to remove the stimulation artifacts using a variation on the average pattern subtraction method (Hashimoto et al., 2002; Bar-Gad et al., 2004). Specifically, a moving average was calculated using a running window, whose size was adjusted to maximize signal quality after artifact removal (typically $30-40$ pulses). The signal was then off-line sorted (OFS-2.8.4, Plexon) to generate one or more spike trains. The spike train's quality and stability were assessed according to the following criteria: $(1)<0.1 \%$ short $(<1 \mathrm{~ms}$ ) interspikeintervals; (2) normalized signal-to-noise ratio (SNR) typically 4-5 (Joshua et al., 2007); (3) spike shape not significantly altered throughout the session.

To explore the dynamics of the neuronal response to stimulation, three periods were defined. The "early period," stimuli 100-1100, demonstrated the initial response and was chosen to avoid biases resulting from large artifacts or full inhibition during the first few tens of stimuli that were evident in some cells. The "late period," stimuli 4000-5000, was typically after the stabilization (see Results) of the response. Finally, the "very-late period," stimuli 15,000-16,000, served as a control for the stabilization.
The following measures of the neuronal response to stimulation were calculated:

Firing rate. Firing rate functions were calculated using blocks of 100 and 1000 stimuli, while compensating for dead time and spike shape length. Significant change in firing rate relative to baseline before stimulation was calculated using bootstrap analysis. For each cell, random intervals in the prestimulation period were chosen in blocks of $5 \mathrm{~s}$, and a population of baseline firing rates was generated by repeating this procedure 100 times. Significant change in firing rate during stimulation was then tested using a $t$ test $(p<0.01)$.

Time-locked response. A time-locked response is defined as an increased probability for firing at a specific latency relative to the stimulus, exhibited as a peak in a peristimulus time-histogram (PSTH). A timelocked response was considered significant if it crossed confidence lines that were constructed from sham-PSTH calculated on baseline activity before stimulation $(p<0.01)$. To explore the evolution of the timelocked response over time, partial PSTHs were calculated for the early and late periods. A peak in the PSTH was defined as the five consecutive bins with the maximal sum over the PSTH, focusing on the central peak at $2-4.5 \mathrm{~ms}$ and $2-6 \mathrm{~ms}$ after the stimulus for the early and late periods, respectively. To enable comparison between cells, we normalized the peak timing and amplitude for each neuron. The peak timing was normalized to a range of $0^{\circ}$ (stimulus onset) to $360^{\circ}$ (end on interval between two consecutive stimuli) after excluding the dead time period, to fit the degrees on a unit circle. The peak amplitude was normalized to the percentage of spikes at the peak $\pm 1 \mathrm{~ms}$, out of the total number of spikes in the PSTH. The degree of temporal locking was assessed using a locking index (LI), such that higher LI values imply a stronger time-locked response. The LI was defined as the root mean square (RMS) of the difference between the PSTH and a sham-PSTH (representing a flat nonlocked response), after smoothing by a semi-Gaussian window ( $\mathrm{SD}=50 \mu \mathrm{s})$.

\section{Results}

HF microstimulation was applied in the GPe and GPi, and neurons from both segments were recorded. The activity of the neurons was analyzed after removal of the stimulation artifact. The removal of artifacts resulted in a complete dead-time (time in which spikes could not be identified at all) of $0.79 \pm 0.24 \mathrm{~ms}$ (mean $\pm \mathrm{SD}, 0.1 \pm 0.03$ of the interstimulus-interval) and an additional partial dead-time (time in which the spike shape was distorted because of its partial masking by the artifact), leading to misidentification of spikes during $0.42 \pm 0.06 \mathrm{~ms}$ of the spike shape length (mean $\pm \mathrm{SD}, 0.05 \pm 0.01$ of the interstimulusinterval). After the removal of the artifacts, the neurons were offline sorted and a total of $122 \mathrm{GPi}$ and $127 \mathrm{GPe}$ ( 85 for monkey A and 164 for monkey N) stable and high-grade neurons were used for this study (see supplemental material 1, available at www.jneurosci.org, for histological reconstruction).

The mean baseline firing rate of GPe and GPi neurons during the prestimulation period was $60.2 \pm 29.7$ and $78.5 \pm 29 \mathrm{sp} / \mathrm{s}$ (mean $\pm \mathrm{SD}$ ), respectively. The mean firing rate of GPi neurons during the whole stimulation time relative to the prestimulation period significantly decreased for both GPe $(-6.8 \pm 1.7 \mathrm{sp} / \mathrm{s}$, mean $\pm \mathrm{SEM})$ and GPi $(-12.2 \pm 2.7 \mathrm{sp} / \mathrm{s}$, mean $\pm \mathrm{SEM}) \mathrm{stim}-$ ulation (paired $t$ test, $p<0.001$ ), whereas the firing rate of GPe neurons remained unchanged (Fig. $1 A$ ). The firing patterns during stimulation consisted of time-locked responses manifested as a peak in the PSTH. The time-locked response was evident in the population firing pattern (Fig. $2 \mathrm{~A}$ ), where GPe neurons tended to fire $3.5 \pm 0.13 \mathrm{~ms}$ (mean $\pm \mathrm{SEM}$ ) after the stimulus, and GPi neurons tended to fire at a significantly longer latency of $4.2 \pm$ $0.12 \mathrm{~ms}$ (mean \pm SEM) after the stimulus ( $t$ test, $p<0.01$ ).

Normalized rate functions were calculated for each neuron over the stimulation period. The primary changes in firing rate occurred during the first $10 \mathrm{~s}$ of stimulation. Sixty-nine percent $(171 / 249)$ of the neurons significantly altered their firing rate 
A

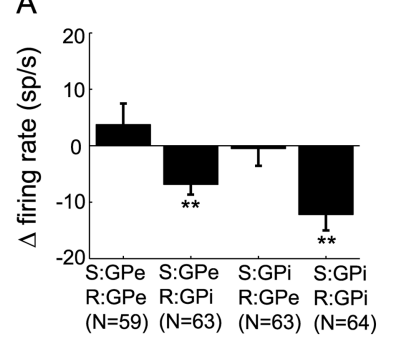

D

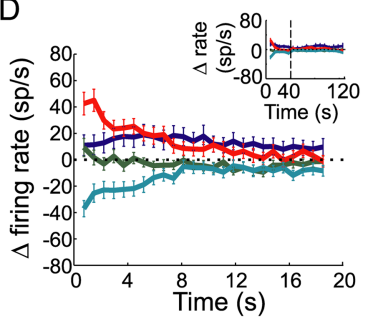

B

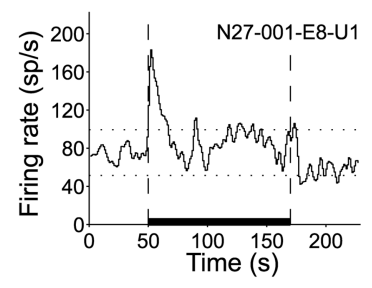

E

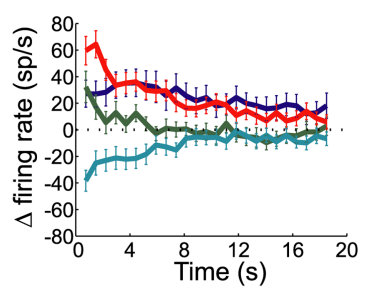

C

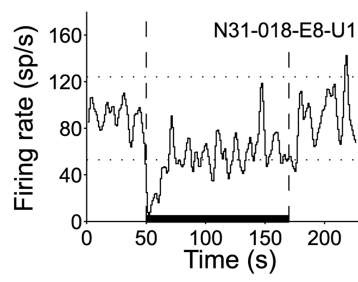

$\mathrm{F}$

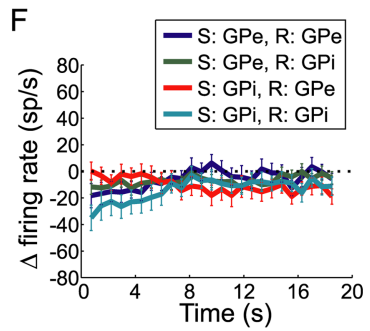

Figure 1. Firing rate changes during stimulation. $A$, Mean firing rate changes of GPe and GPi neurons during stimulation compared with the prestimulation period ( $\pm S E M,{ }^{* *} p<0.001$, paired $t$ test). S, Stimulation site; $R$, recording site. $B, C$, Examples of $(\boldsymbol{B})$ a GPe neuron with increased firing rate and $(\boldsymbol{C})$ a GPi neuron with decreased firing rate at the beginning of GPi stimulation For $\boldsymbol{B}$ and $\boldsymbol{C}$, thick horizontal line marks the stimulation period, dashed vertical lines mark stimulation beginning and end, and dotted horizontal lines mark confidence limits $(p<0.01)$. Firing rate is calculated using $1 \mathrm{~s}$ bins, smoothed by a Gaussian window, $S D=0.8 \mathrm{~s}$. D, Firing rate change during the first $20 \mathrm{~s}$ of stimulation compared with prestimulation baseline (mean \pm $S E M)$. Inset, Firing rate change during the whole stimulation period. Data from two monkeys are shown to the left of the vertica dashed line, data from monkey $N$ only are shown to the right of the dashed line (longer stimulation time). $\boldsymbol{E}, \boldsymbol{F}$, Same as $\boldsymbol{D}$, but for cells that had time-locked response to stimulation $(\boldsymbol{E})$ and cells that were not time-locked to stimulation $(\boldsymbol{F})$ during the early period. In color coding: $S$, stimulation site; $R$, recording site.

during the early period (stimuli 100-1100), but these percentages were significantly smaller during the late period (stimuli 4000 $5000)\left[53 \%(131 / 249), \chi^{2}\right.$ test, $p<0.05$ (Table 1$\left.)\right]$. The proportion of increasing and decreasing neurons varied based on the location (nucleus) of both the stimulating and recording electrodes (Table 1, see examples in Fig. $1 B, C$ ). During the early period, the percentage of GPe neurons that increased their rate was significantly larger than the percentage of neurons that decreased their rate $\left(\chi^{2}\right.$ test, $\left.p<0.01\right)$. This trend was reversed in the GPi, where a significantly larger proportion of cells decreased their rate $\left(\chi^{2}\right.$ test, $\left.p<0.05\right)$. Changes in firing rate were larger during the early period compared with the late period, where the firing rate of GPe cells significantly increased, and the firing rate of GPi cells significantly decreased ( $t$ test, $p<0.001$ ). The latter effect was significantly larger during GPi stimulation compared with GPe stimulation $(t$ test, $p<0.001)$ (Fig. $1 D$ ). Further analysis revealed two populations of cells: (1) responding neurons, which both changed their firing rates dramatically and had timelocked responses (Fig. 1E), and (2) nonresponding neurons, which were neither time locked to stimulation nor changed their rate (Fig. $1 F$ ).

PSTHs and partial PSTHs during the early and late stimulation periods were constructed. Most of the recorded neurons were significantly time locked to the stimulation pulses during the early period $(61 \%, 153 / 249)$, but the percentages of timelocked neurons were significantly reduced during the late period [32\% (80/249), $\chi^{2}$ test, $p<0.01$ (Table 1)]. The response patterns consisted of one or more peaks in the PSTH, mostly with a central peak at $2-4.5 \mathrm{~ms}$ after the stimulus ( $89 \%$ of neurons during the early period, 136/153), but also at $4.5-7.4 \mathrm{~ms}(53 \%, 81 / 153)$ and $0-2 \mathrm{~ms}(14 \%, 22 / 153)$ after the stimulus. The time-locked responses changed over time (example in Fig. $2 B$ ). In $43 \%$ of the responding neurons (58/136), the time-locked response com-

pletely faded away after $30 \mathrm{~s}$. In the remaining cells, the PSTH peak timing significantly shifted away from the stimulus onset (mean \pm SD: $0.34 \pm 0.46 \mathrm{~ms}, t$ test, $p<0.001$ ), and the PSTH peak amplitude was significantly reduced (mean $\pm \mathrm{SD}$ : $73 \pm 102 \mathrm{sp} / \mathrm{s}$ reduction, $t$ test, $p<0.001$ ). After $30 \mathrm{~s}$, the time-locked response stabilized and reached a steady state. No significant differences of peak amplitude and latency were evident between the late period and the very-late period (stimuli 15,000$16,000)$ (paired $t$ test, $p>0.05$ ) (example in Fig. $2 B$ ). The normalized peak time and amplitude were calculated for each neuron for the early (Fig. 2C) and late (Fig. 2D) responses. The latency of the normalized peak time was longer during the late period compared with the early one. The normalized amplitude indicated that the response around the peak was smaller during the late period. A LI was calculated, such that higher LI values indicate a stronger time-locked response. The LI during the late period was smaller compared with the early period in most cells (Fig. $2 E$ ), indicating a decay of the time-locked response over time. Of the neurons that showed an evolving time-locked response to stimulation, 19 were recorded during a second session of stimulation after a 12-40 min off-stimulation period. The decay of the response was fully reversible in all the neurons: once HFS was resumed, the response was set back to its initial level, as reflected in both peak amplitude and timing (Fig. $3)$.

\section{Discussion}

In this study we showed that HF microstimulation affects both the firing rate and pattern of neuronal activity. Two populations of neurons emerged: responding neurons that were affected by the stimulation and displayed both a time-locked response and a change in firing rate, and nonresponding neurons that displayed neither. Moreover, our results demonstrate the dynamic nature of the neuronal response to stimulation and reveal the modification of BG network information transmission during HFS in vivo. The effects of stimulation evolved over time, during which the primary changes were apparent in the first $10 \mathrm{~s}$ of stimulation. Initially, the firing rates of the responding neurons were significantly altered: GPi cells tended to decrease their rate and GPe cells tended to increase their rate, primarily but not exclusively during GPi stimulation. The time-locked response was stronger at the beginning of the stimulation and tended to weaken as the stimulation progressed: the peak of the response shifted away from the stimulus and its amplitude decreased. In many cells the timelocked response completely faded away. The neuronal response to stimulation reached a steady state after $30 \mathrm{~s}$, and was then stable for the rest of the stimulation period.

Time-locked responses predominated in a large fraction of the recorded cells. Other studies have reported peristimulus peaks of activity with comparable latencies (Hashimoto et al., 2003; BarGad et al., 2004; Kita et al., 2005; McCairn and Turner, 2009). Our data elaborate on the characteristics of these time-locked responses: most of the cells tended to fire at a latency of $2-4.5 \mathrm{~ms}$ 


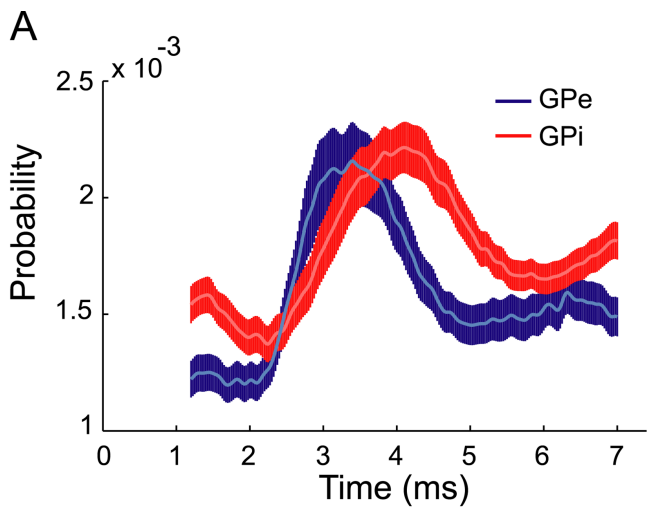

B
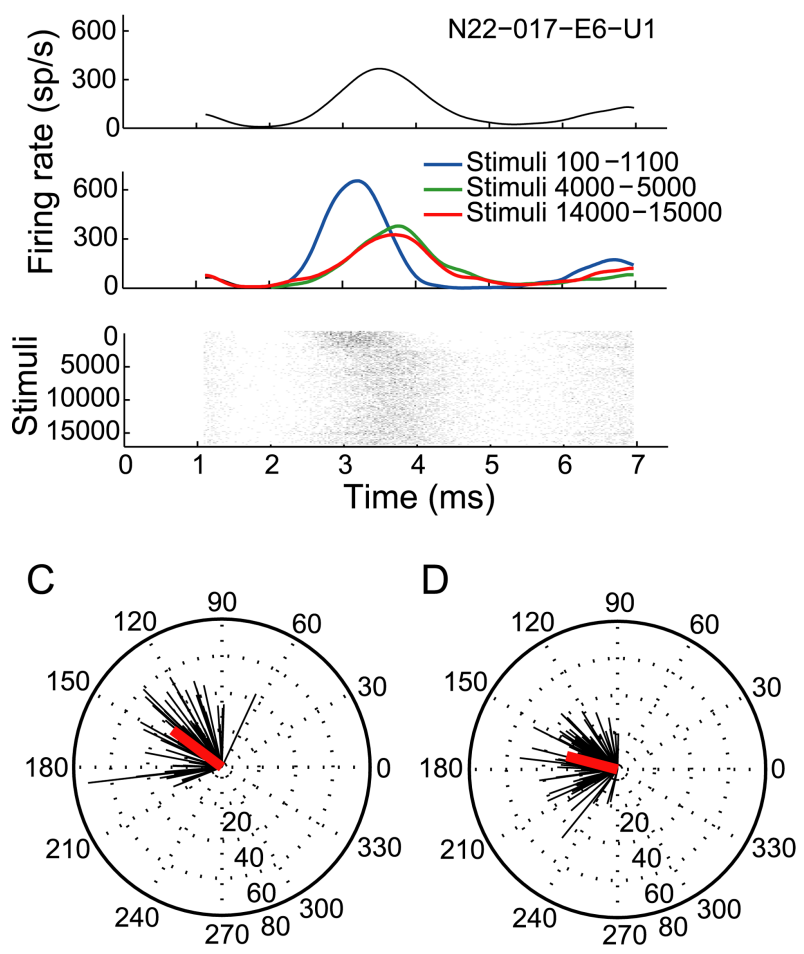

E

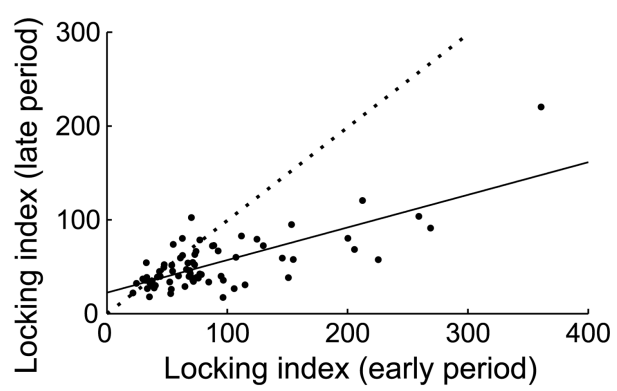

Figure 2. Time-locked response changes during stimulation. A, Population PSTH of all GPe (blue) and GPi (red) neurons during stimulation ( \pm SEM, smoothed by a Gaussian window, $S D=50 \mu \mathrm{s})$. $\boldsymbol{B}$, Example of dynamic time-locked response of a GPe neuron during GPe stimulation. Top, PSTH of response during the whole stimulation period. Middle, Partial PSTHs for different periods. Bottom, Raster plot smoothed over 100 consecutive stimuli. C, D, Compass plots of normalized peaks and amplitudes of PSTHs during the early $(\boldsymbol{C})$ and late $(\boldsymbol{D})$ periods. Each neuron is represented by one vector: the direction represents the normalized peak time and the length represents the normalized peak amplitude. The red line represents the mean normalized response. $\boldsymbol{E}$, Locking index during early versus late periods. Dotted line at diagonal, linear regression is shown as a solid line $\left(R^{2}=0.55, p<0.001\right)$. after the stimulus, a response which is likely mediated by synaptic transmission (Hammond et al., 2008). A possible mechanism that may underlie these effects is activation of STN excitatory efferent fibers through a monosynaptic connection (Hammond et al., 2008), which then leads to time-locked activity in the GP. When stimulating in the GPe and recording in the GPi, and vice versa, the excitation phase might be caused by activation of STN efferent fibers with collaterals in both GPe and GPi (Parent et al., 2000). Other possible mechanisms include direct activation of efferent and afferent fibers with a broader effect on other BG nuclei, which are massively interconnected (Hammond et al., 2008; Johnson and McIntyre, 2008).

The evolution of the time-locked response provides further insights into the mechanism of stimulation. As the stimulation progressed, the response to stimulation went through a decay process in both firing rate change and time-locked response. The time-locked response shifted in time and its amplitude was reduced, implying a short-term (reversible) depression of the affected synapses (Hanson and Jaeger, 2002; Rav-Acha et al., 2005; McCairn and Turner, 2009) linking the stimulated fibers and the recorded neurons. Although further in vitro studies are required to explore the synaptic mechanism of stimulation, we suggest a possible mechanism underlying this depression. The amount of vesicles available for release in the affected synapses might be reduced as the stimulation progresses because of the fast recurrent activation and neurotransmitter depletion in the synapses (Markram, 1997; McIntyre et al., 2004), resulting in a slower buildup of the poststimulus response (supplemental material 3, available at www.jneurosci.org). The slower accumulation of input required for spike generation, in turn, leads to: (1) a reduction in the total number of generated spikes by the postsynaptic (recorded) neuron, and (2) a longer latency relative to the stimulus, with a larger variability of latencies. This is manifested in our data as an overall weaker response to stimulation, with a widening of the peak in the PSTH and lengthening of its latency. However, some information transmission is preserved during stimulation because not all neurons are activated; thus, some of the synapses are not depressed. We conducted a neural network simulation of the reduction in synaptic efficacy and its effect on the firing pattern of postsynaptic neurons. The simulation resulted in a dynamic time-locked response similar to the one observed in the recorded neurons (supplemental material 3, available at www.jneurosci.org). Still, it is possible that other nonsynaptic mechanisms might be involved in the response depression, such as network dynamics (Johnson and McIntyre, 2008) or alterations of membrane conductance as a result of changes in extracellular concentrations (Bellinger et al., 2008).

Relating the dynamic changes observed in our study to the clinical effects of DBS requires addressing some differences: First, the effects of macrostimulation are expected to be more pronounced because a larger volume of tissue is affected by stimulation, resulting in a larger fraction of depressed synapses. Second, our stimulation intensities were below behavioral threshold; therefore, we cannot directly relate the synaptic depression to amelioration of clinical symptoms. Third, the effects of stimulation in other deep brain areas (such as the STN and thalamus) or during other normal or pathological states may also follow such dynamic changes. However, different cellular or even state (such as dopamine levels) properties may yield different accommodation processes (Prescott et al., 2009).

Our results support the hypothesis that short-term synaptic plasticity is involved in the HFS mechanism. The synaptic changes at the single cell level may disrupt the balance of com- 
Table 1. Firing rate modulation and time-locked responses during stimulation

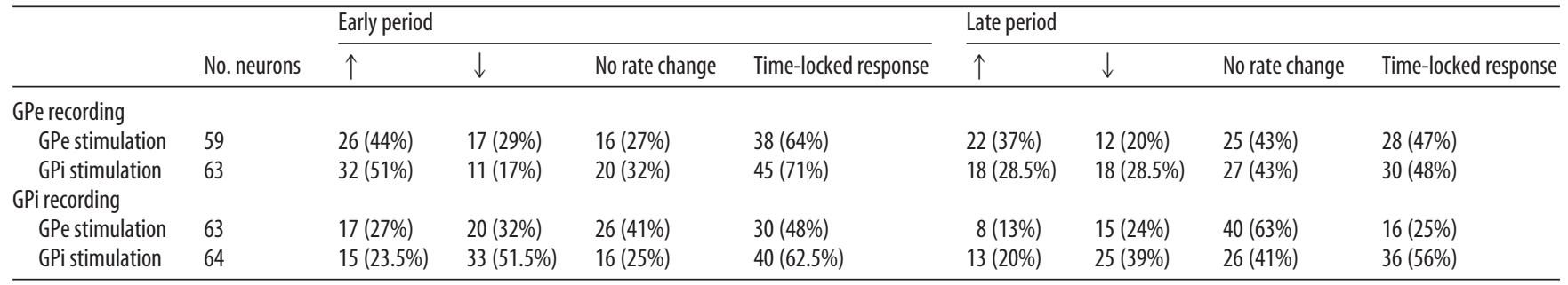

$\uparrow$, Significant rate increase; $\downarrow$, significant rate decrease.

A
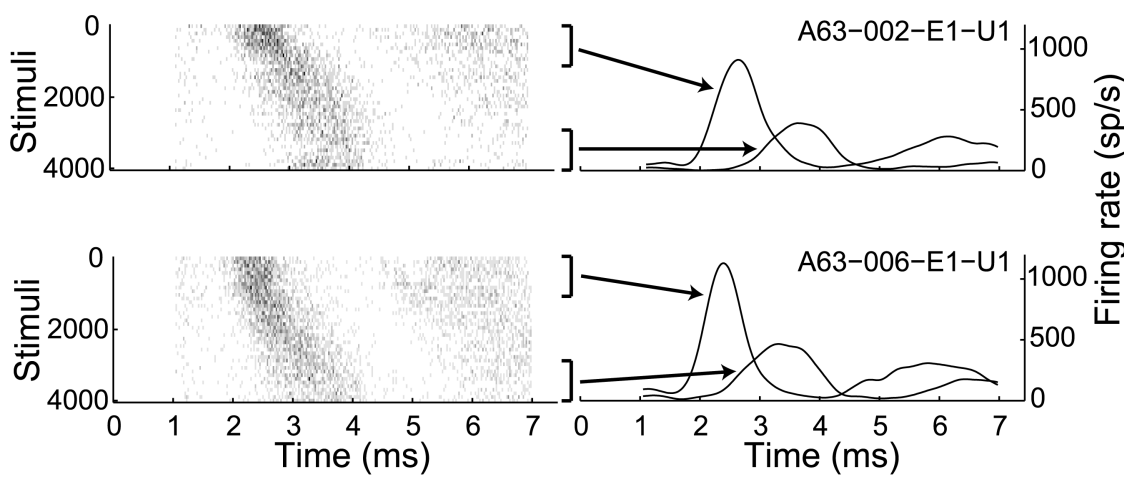

B

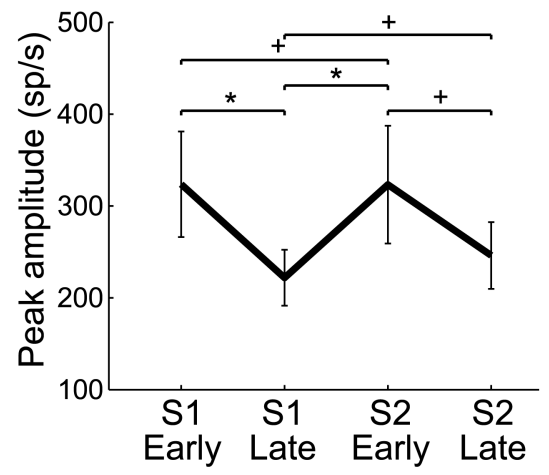

C

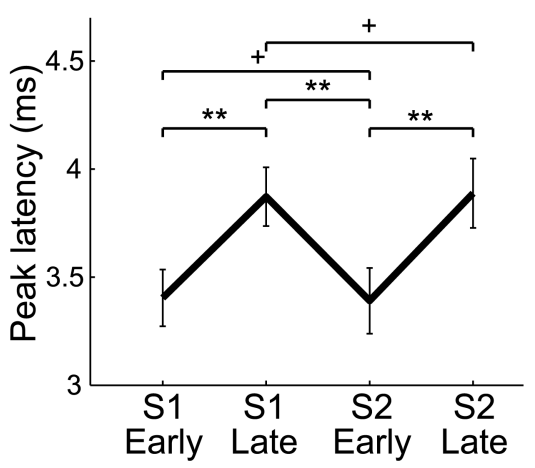

Figure 3. Reversibility of time-locked response to stimulation. $A$, Example of the reversible nature of the time-locked response of a GPe neuron during two sessions of GPi stimulation (top, first session; bottom, second session). Left, Raster plots of the two sessions. Right, Partial PSTHs of the first and last 1000 pulses of each stimulation session. $\boldsymbol{B}, \boldsymbol{C}$, Reversibility of time-locked response to stimulation during the second session compared with the first session as reflected in the peak amplitude $(\boldsymbol{B})$ and latency (C). Error bars indicate SEM; S1 and S2, first and second session, respectively. ${ }^{*} p<0.05,{ }^{* *} p<0.01,{ }^{+} p>0.05$.
Dorval AD, Russo GS, Hashimoto T, Xu W, Grill WM, Vitek JL (2008) Deep brain stimulation reduces neuronal entropy in the MPTPprimate model of Parkinson's disease. J Neurophysiol 100:2807-2818.

Dostrovsky JO, Levy R, Wu JP, Hutchison WD, Tasker RR, Lozano AM (2000) Microstimulationinduced inhibition of neuronal firing in human globus pallidus. J Neurophysiol 84:570-574.

Filali M, Hutchison WD, Palter VN, Lozano AM, Dostrovsky JO (2004) Stimulation-induced inhibition of neuronal firing in human subthalamic nucleus. Exp Brain Res 156:274-281.

Hahn PJ, Russo GS, Hashimoto T, Miocinovic S, Xu W, McIntyre CC, Vitek JL (2008) Pallidal burst activity during therapeutic deep brain stimulation. Exp Neurol 211:243-251.

Halpern C, Hurtig H, Jaggi J, Grossman M, Won M, Baltuch G (2007) Deep brain stimulation in neurologic disorders. Parkinsonism Relat Disord 13:1-16.

Hammond C, Ammari R, Bioulac B, Garcia L (2008) Latest view on the mechanism of action of deep brain stimulation. Mov Disord 23:2111-2121.

Hanson JE, Jaeger D (2002) Short-term plasticity shapes the response to simulated normal and parkinsonian input patterns in the globus pallidus. J Neurosci 22:5164-5172.

Hashimoto T, Elder CM, Vitek JL (2002) A template subtraction method for stimulus artifact removal in high-frequency deep brain stimulation. J Neurosci Methods 113:181-186.

Hashimoto T, Elder CM, Okun MS, Patrick SK, Vitek JL (2003) Stimulation of the subthalamic nucleus changes the firing pattern of pallidal neurons. J Neurosci 23:1916-1923.

Johnson MD, McIntyre CC (2008) Quantifying the neural elements activated and inhibited by globus pallidus deep brain stimulation. J Neurophysiol 100:2549-2563.

munication at the network level, resulting in an interruption in the abnormal information flow. Therefore, depression of synaptic transmission may play an important role in generating a stimulation-based steady state that interferes with pathological information processing and transmission during the parkinsonian state.

\section{References}

Bar-Gad I, Elias S, Vaadia E, Bergman H (2004) Complex locking rather than complete cessation of neuronal activity in the globus pallidus of a 1-methyl-4-phenyl-1,2,3,6-tetrahydropyridine-treated primate in response to pallidal microstimulation. J Neurosci 24:7410-7419.

Bellinger SC, Miyazawa G, Steinmetz PN (2008) Submyelin potassium accumulation may functionally block subsets of local axons during deep brain stimulation: a modeling study. J Neural Eng 5:263-274.

Boraud T, Bezard E, Bioulac B, Gross C (1996) High frequency stimulation of the internal Globus Pallidus (GPi) simultaneously improves parkinsonian symptoms and reduces the firing frequency of GPi neurons in the MPTP-treated monkey. Neurosci Lett 215:17-20.
Joshua M, Elias S, Levine O, Bergman H (2007) Quantifying the isolation quality of extracellularly recorded action potentials. J Neurosci Methods 163:267-282.

Kita H, Tachibana Y, Nambu A, Chiken S (2005) Balance of monosynaptic excitatory and disynaptic inhibitory responses of the globus pallidus induced after stimulation of the subthalamic nucleus in the monkey. J Neurosci 25:8611-8619.

Markram H (1997) A network of tufted layer 5 pyramidal neurons. Cereb Cortex 7:523-533.

McCairn KW, Turner RS (2009) Deep brain stimulation of the globus pallidus internus in the parkinsonian primate: local entrainment and suppression of low frequency oscillations. J Neurophysiol 101:1941-1960.

McIntyre CC, Savasta M, Kerkerian-Le Goff L, Vitek JL (2004) Uncovering the mechanism(s) of action of deep brain stimulation: activation, inhibition, or both. Clin Neurophysiol 115:1239-1248.

Meissner W, Leblois A, Hansel D, Bioulac B, Gross CE, Benazzouz A, Boraud $\mathrm{T}$ (2005) Subthalamic high frequency stimulation resets subthalamic firing and reduces abnormal oscillations. Brain 128:2372-2382. 
Montgomery EB Jr (2006) Effects of GPi stimulation on human thalamic neuronal activity. Clin Neurophysiol 117:2691-2702.

Parent A, Sato F, Wu Y, Gauthier J, Lévesque M, Parent M (2000) Organization of the basal ganglia: the importance of axonal collateralization. Trends Neurosci 23:S20-S27.

Prescott IA, Dostrovsky JO, Moro E, Hodaie M, Lozano AM, Hutchison WD (2009) Levodopa enhances synaptic plasticity in the substantia nigra pars reticulata of Parkinson's disease patients. Brain 132:309-318.

Rav-Acha M, Sagiv N, Segev I, Bergman H, Yarom Y (2005) Dynamic and spatial features of the inhibitory pallidal GABAergic synapses. Neuroscience 135:791-802.

Rodriguez-Oroz MC, Obeso JA, Lang AE, Houeto JL, Pollak P, Rehncrona S, Kulisevsky J, Albanese A, Volkmann J, Hariz MI, Quinn NP, Speelman
JD, Guridi J, Zamarbide I, Gironell A, Molet J, Pascual-Sedano B, Pidoux B, Bonnet AM, Agid Y, et al. (2005) Bilateral deep brain stimulation in Parkinson's disease: a multicentre study with 4 years follow-up. Brain 128:2240-2249.

Schneider JS, Gonczi H, Decamp E (2003) Development of levodopainduced dyskinesias in parkinsonian monkeys may depend upon rate of symptom onset and/or duration of symptoms. Brain Res 990: $38-44$.

Szabo J, Cowan WM (1984) A stereotaxic atlas of the brain of the cynomolgus monkey (Macaca fascicularis). J Comp Neurol 222:265-300.

Xu W, Russo GS, Hashimoto T, Zhang J, Vitek JL (2008) Subthalamic nucleus stimulation modulates thalamic neuronal activity. J Neurosci 28 : $11916-11924$. 